\title{
The Impact of a Character Posture Model on the Communication of Affect in an Immersive Virtual Environment
}

\author{
Vinoba Vinayagamoorthy, Anthony Steed, Member, IEEE, and Mel Slater
}

\begin{abstract}
This paper presents the quantitative and qualitative findings from an experiment designed to evaluate a developing model of affective postures for full-body virtual characters in immersive virtual environments (IVEs). Forty-nine participants were each requested to explore a virtual environment by asking two virtual characters for instructions. The participants used a CAVE-like system to explore the environment. Participant responses and their impression of the virtual characters were evaluated through a wide variety of both quantitative and qualitative methods. Combining a controlled experimental approach with various data-collection methods provided a number of advantages such as providing a reason to the quantitative results. The quantitative results indicate that posture plays an important role in the communication of affect by virtual characters. The qualitative findings indicated that participants attribute a variety of psychological states to the behavioral cues displayed by virtual characters. In addition, participants tended to interpret the social context portrayed by the virtual characters in a holistic manner. This suggests that one aspect of the virtual scene colors the perception of the whole social context portrayed by the virtual characters. We conclude by discussing the importance of designing holistically congruent virtual characters especially in immersive settings.
\end{abstract}

Index Terms—Virtual characters, nonverbal behavior, posture, facial expression presence, physiology, evaluation, virtual reality.

\section{INTRODUCTION}

$\mathrm{T}$ HIS paper presents an experiment designed to evaluate the responses of participants to sequences of affective behaviors exhibited by humanoid virtual characters in an immersive virtual environment (IVE). We investigate three main topics. The first is whether responses of participants to the underlying emotion portrayed by a virtual character are in keeping with those observed in the physical world. For instance, if a participant encounters a virtual character exhibiting postures indicative of anger, does the participant respond accordingly and which cues play a more important role in instigating the response? The second topic explores the range and variation of participant responses to affective virtual characters exhibiting minimal behavioral cues. In addition to these two main issues, we also describe the different methods that we used to evaluate a model of a behavioral cue such as the postural model that we used in animating the virtual characters since it is difficult to gauge

- V. Vinayagamoorthy is with the British Broadcasting Corporation (BBC), BBC Research and Innovation, B14, Kingswood Warren, Tadworth, Surrey KT20 6NP UK. E-mail: vinoba.vinayagamoorthy@bbc.co.uk.

- A. Steed is with the Department of Computer Science, University College London, Gower Street, London WC1E 6BT UK.

E-mail: anthony.steed@cs.ucl.ac.uk.

- M. Slater is with the Department of Computer Science, University College London, Gower Street, London WC1E 6BT UK and ICREA-Universitat Politècnica de Catalunya (UPC), Centre de Realitat Virtual (CRV), Edificio U, C/ Llorens i Artigas 4-6 08028 Barcelona, Spain.

E-mail:melslater@gmail.com.

Manuscript received 10 Oct. 2007; revised 31 Jan. 2008; accepted 11 Feb. 2008; published online 10 Apr. 2008.

Recommended for acceptance by M.C. Lin.

For information on obtaining reprints of this article, please send e-mail to: tvcg@computer.org, and reference IEEECS Log Number

TVCG-2007-10-0159.

Digital Object Identifier no. 10.1109/TVCG.2008.62.

1077-2626/08/\$25.00 @ 2008 IEEE this with a single measure. A combination of psychophysiological instruments, measures of spatial behavior, presence questionnaires, and postexperimental interviews were used to collect the participant's responses to the virtual characters. We argue that a combination of these different types of measures is essential in understanding such a complex phenomenon.

Virtual environments without some depiction of human activity can be unrealistic and uninteresting. Moreover, some virtual reality (VR) applications demand interaction with virtual characters such as in games [1], training environments [2], and virtual therapy [3]. A virtual character can harness the users' automatic responses to the human form and behavior, and thereby allow VR users to achieve a kind of empathic interaction that would otherwise be difficult. However, creating an interactive and responsive expressive virtual character is difficult because of the complex nature of human nonverbal behavior such as facial expression, body posture, and gestures. Although a lot is known about the forms of these behaviors, there is surprisingly little research on models that generate affective behavior across all modalities such as postures and gestures [4], [5], [6]. Even less is known about participant responses to expressive virtual characters. Therefore, the first issue encountered is to be able to identify common attributes that can be used to build a model of affective behavioral cues. The second issue revolves around the methods used in evaluating a model of behavioral cues.

In this paper, we focus on two types of behavioral cues (Posture and Facial expression) and two emotional states (anger and sadness). This work builds on the methods used in [7] to model behavioral cues based on observations 
derived from previous work. In Section 2, we review relevant background work on posture and its role in the communication of affect. We integrate results obtained in a nonimmersive study, where static postures were categorized based on specific emotion labels [4], into a model used to control the perceived behavior of a virtual character in an immersive setting. The model was used in an experiment designed to explore the relative importance of posture in comparison with facial expressions in the communication of an underlying emotional state. We give a description of the experimental goals in Section 3. Full details of the experimental setup and a description of the method of analysis are reported in the subsequent section (Section 4). A preliminary analysis of the participant responses collected through questionnaires in the experiment was presented in [8]. We present an analysis of both subjective and objective participant responses collected in the experiment (Sections 5 and 6). In addition, we investigate an approach to designing expressive virtual characters based on an approach to examine a wide range of participant responses to virtual sensory data [9]. This was addressed through the post hoc analysis of the contribution of the different methods of data collection and analysis employed in the design of the experiment (Section 6).

\section{BACKGROUND}

The creation of an interactive and responsive virtual character in real-time applications is a complex and timeconsuming task. Users of such applications have specific expectations of how people behave and respond given a situation, and therefore, generally expect humanoid virtual characters to behave in a manner befitting their appearance and will often be disturbed by discrepancies in such behavior. In fact, it has been argued that the importance of behavior fidelity of the virtual character far outweighs the importance of visual realism in some applications [10].

Research related to the design of behaviors in virtual characters can be broadly grouped into two distinct but interrelated themes. The first deals with the generation of a virtual character's internal state through computational models that simulate the dynamics of psychological states such as emotions [11], [12]. An exhaustive review of existing generative models of personality and emotion is available in [6]. The second theme deals with the representation of the virtual characters' internal state through observable behavioral cues, which remains largely unexplored. This presents problems when trying to computationally model the representation of internal states through consistent behavioral cues in full-body virtual characters. Section 2.1 reviews the previous work related to the representation of emotions in virtual characters, while Section 2.2 briefly presents the various methods in which virtual characters have been evaluated.

\subsection{Representing Emotions in Virtual Characters}

In people's efforts to interpret or evaluate the motives, moods, and behaviors of others, they look to a combination of postural cues as well as facial expression and speech [13]. It is part of the conversational feedback process that adds richness and versatility to social interaction [14]. The study of behavioral cues of affect has been heavily biased toward facial expressions [5] since it is the most closely observed behavioral cue [15]. There is also evidence that there is some agreement when it comes to recognition of affect facial expressions across different cultures [16]. This has led to a number of systems capable of producing affective facial expressions through using a set of universally recognized facial expressions [17] and combinations of these facial expressions [12], [18].

Unlike research on facial expressions, the role of the body in the expression of affect is still debatable. Some researchers argue that the body conveys the degree of intensity of an emotional state [19]. Others have argued that postures can be a dominant source of cues in the perception of emotions [20], [21] and, in some cases, an equally accurate source of cues to an emotion as face expressions [22]. For instance, it has been argued that even though the face is the most expressive area of the body and the primary carrier of affect, when it is accompanied by congruent bodily cues there is less ambiguity in communicating affect [20], [23], [24]. Postural cues are also thought to be of more importance when facial expressions are not visible [25]. Furthermore, an individual may display postural cues without the conscious intention to communicate affect. The display of emotive facial expressions may be toned down for a particular social context, however, accompanying bodily cues help decrease ambiguity in the communication [21]. This is because individuals pay less conscious attention to the control of posture than facial expressions [21]. Since there are no empirical studies regarding the attributes of posture, this poses a technical challenge in trying to build a model of affective postures.

In early work, James suggested that the postures of some parts of the body were more important in expressing specific emotions [26]. In keeping with this work, Wallbott was able to single out distinct behavioral cues specific to certain emotions [27]. Recently, De Silva and BianchiBerthouze collected motion data containing affective gestures from actors trained to portray an underlying emotion with the intention of measuring the salient features in the data and using it to discriminate between a set of emotions [28]. Their findings highlighted the importance of posture to overall communication of affect, suggesting that postural cues can be used to communicate affect effectively. However, their model did not discriminate sufficiently between some emotional states due to a lack of sufficient data. More recently, Kelsmith et al. conducted a statistical analysis of emotional postures produced by Japanese actors and identified three main dimensions that explained the variation of the postures [29]. However, their model is quite new and has not been adapted to exclude cultural dependencies.

Another study is of direct relevance to this paper. Coulson designed a set of static postures designed in keeping with previous theoretical work [4]. The study was limited to examining postures associated with six emotions: anger, disgust, fear, happiness, sadness, and surprise. Coulson's findings mirrored those of [28], in that specific postures were attributed to an emotional state especially in the cases of anger, sadness, and happiness. Based on the 
findings, Coulson reported on the common attributes shared by postures in a given affective group [4]. Even though the work carried out by Coulson was limited to a small number of emotion labels in a nonimmersive setting, the resultant postures gives a starting point with which to create a postural behavior model. The model of posture (Section 4.8.2) used in our experiment is based on the work reported by Coulson [4].

\subsection{Evaluating Affective Virtual Characters}

The effectiveness of a model of behavioral cues in virtual characters can be measured with respect to the extent to which participants act and respond to virtual sense data as if it were real [2], [9]. This is an operationalization of the concept usually referred to as presence in VEs that avoids sole reliance on questionnaire to understand responses. The sole usage of questionnaires in collecting participant responses has been criticized due to its dependencies on the participant's accurate post hoc recall, processing, and rationalization of the experience [30]. Other quantitative but more objective means of measuring participant responses include analysis of spatial behavior [31], interpersonal measures such as flow of conversation [2], and psychophysiological responses [32], [33]. This is not to say that such objective data rules out the use of or is more valuable than questionnaire or interview-based data. Combining a controlled laboratory-based experimental approach with various data-collection methods provides a number of advantages. These include reinforcement of any results with the addition of a reason to any quantitative results, the possibility of revealing a reason for contradictory results, the potential discovery of new avenues for research, and validating the aforementioned advantages of using a combined research methodology. In this paper, we use a combination of methods to understand participant responses to the virtual characters.

\section{Experiment Goals and Hypothesis}

As covered in the previous section, to date, the existing and limited studies on postures have been conducted in nonimmersive settings and in a static form. In addition, the role of posture in a given social context has not been investigated with respect to virtual characters.

The goal of our experiment was threefold. First, we were interested in exploring the relative importance of the roles played by facial and postural cues in the communication of two emotions (anger and sadness) in a specific social context. We investigate the importance of posture in a situation where the affective state is directed at a focus other than the participant. Second, we examine whether the angry and sad postures reported by Coulson [4] were effective in communicating the desired affect in an immersive setting. Finally, we explore the variations in participant responses to characters perceived to be in a certain emotional state using mixed methods in order to 1) gain insights into improving the developing model of affective posture and 2) generate hypothesis for future studies.

The overall goal of our research has been to better understand the impact of the behavior of virtual characters on people within an IVE, concentrating in particular on postural aspects. We investigate this through the analysis of a range of participant responses collected using mixed methods (Section 1). Our main hypothesis is that adding affective behavioral cues to virtual characters would add to the character's perceived realism. We expect participants to recognize the character's underlying affective state more accurately if the character portrays congruent facial expressions and postural cues. In other words, we anticipate that participants will use posture as a significant cue to interpret the virtual character's affective state.

\section{Experiment Design}

The experiment was designed to explore how participants interpreted and responded to virtual characters designed to be in a specific emotional state. The virtual characters were designed to use two observable behavioral cues (Posture and Facial expression) to represent two emotional states (anger and sadness). Two virtual characters were then presented as part of a scenario to participants in an immersive setting. The following sections give a description of the full experimental design and the apparatus setup.

\subsection{The Scenario}

A scenario was developed in which participants could observe two virtual characters having a discussion in a virtual environment. The verbal content of the discussion was designed to be muffled (unintelligible), in order to ensure that the affective states of the virtual characters could only be inferred through the nonverbal behavioral cues displayed by the characters. Throughout this paper, we refer to one character as the active character and the other as the passive character. The active character displayed an underlying affective state (anger or sadness) through facial and/or postural cues toward the passive character. The passive character portrayed exactly the same "neutral" state throughout the experiment by displaying behavioral cues that were designed to be nonaffective.

Participants were told that the experiment involved interacting with virtual characters in order to obtain directions to explore a virtual maze. The participant was instructed to move toward the characters, and once the participant got close enough to breach the social interpersonal distance as defined by Hall [34], the active character stopped the discussion and adopted a neutral state as well. The two virtual characters then turned toward the participant and interacted with the participant through the use of neutral nonverbal behaviors and verbal content. This meant that the participant played two roles in the experiment: the role of an observer to an emotionally charged discussion between two virtual characters followed by the role of a conversant in a direct face-to-face neutral interaction with the characters (Fig. 1). For the main purposes of this experiment, we were mainly interested in the participants' responses while they played the role of an observer.

This scenario was developed for two reasons. First, it allowed participants to observe and form impressions about the perceived affect of the virtual characters without being explicitly instructed to do so. Second, the scenario designed 


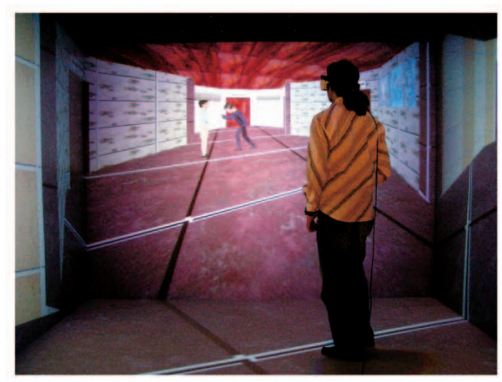

(a)

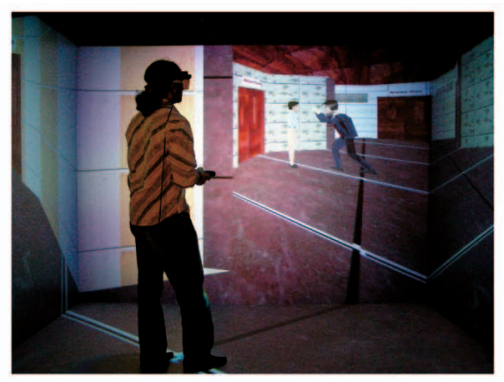

(b)

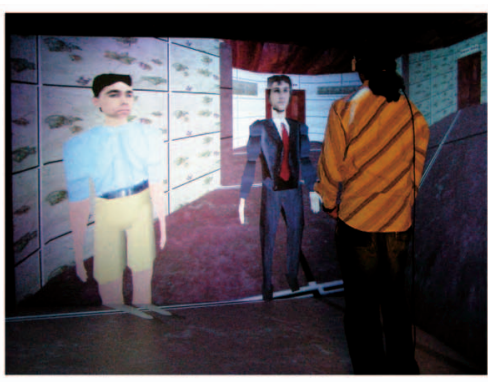

(c)

Fig. 1. Participants' roles in the experiment. (a) Participant while initially observing the characters. (b) Participant still observing. (c) Participant in a direct face-to-face interaction.

for the experiment allowed the participant to observe the affective behavioral cues of a virtual character but only when it was being directed toward another character. This meant that the scenario could be presented to all the participants in a relatively similar manner through the study.

\subsection{Justification of Emotion Choices}

The two emotional states explored in this experiment were anger and sadness. The main emotional state of interest in our research was anger due to its association with threat perception and the varieties of possible responses evoked in individuals witnessing an act of aggression. Anger is seen as a negative emotion; therefore, there is evidence that others focus a heightened attention on individuals displaying the cues to portray anger [35]. Sadness was chosen as a second emotion in order to disambiguate between any possible effects caused by simply having meaningful behavioral cues. Either fear or happiness would have been good choices for the second emotion; however, they both had to be excluded from the study since they could not be portrayed unambiguously using the set of empirically evaluated postures presented in [4].

\subsection{Independent Factors}

Two sets of between-groups 2 (facial expression) $\times 2$ (postural cues) factorial designs were employed in the experiment -one set per emotional state investigated (anger and sadness). As can be observed from Table 1, the two factors in each set control the manner in which the underlying emotional state of the active character is portrayed. In essence, the affective state of the active character toward the passive character was designed to be angry, sad, or neutral

TABLE 1

$2 \times 2$ Factorial Designs: Angry and Sad

\begin{tabular}{|l|c|c||c|c|}
\hline & \multicolumn{2}{|c||}{ Angry } & \multicolumn{2}{c|}{ Sad } \\
\cline { 2 - 5 } & $\begin{array}{c}\text { Emotional } \\
\text { Face }\end{array}$ & $\begin{array}{c}\text { Neutral } \\
\text { Face }\end{array}$ & $\begin{array}{c}\text { Emotional } \\
\text { Face }\end{array}$ & $\begin{array}{c}\text { Neutral } \\
\text { Face }\end{array}$ \\
\hline $\begin{array}{l}\text { Emotional } \\
\text { Posture }\end{array}$ & 7 & 7 & 7 & 7 \\
\hline $\begin{array}{l}\text { Neutral } \\
\text { Posture }\end{array}$ & 7 & 7 & 7 & - \\
\hline
\end{tabular}

The table shows the number of participants assigned to each cell in the design. depending on the behavioral cues used. This created eight conditions; however, the control condition (neutral posture with neutral facial expression) did not need to be repeated for both Angry and Sad, since it is the same in both cases. This results in three emotional states (angry, sad, or neutral) for the active character and just seven distinct conditions.

\subsection{Population}

The participants were recruited through poster, email, and/ or online campaigns through the university campus. They were paid the equivalent of $\$ 10.00$ for an hour-and-half study. In order to eliminate results that were dependent on gender, only male participants were recruited to take part in the experiment. Forty-nine participants were assigned randomly to one of the seven conditions. Most of the participants were in their 20s and more than 85 percent of the participants had very low experience with VR systems. About 43 percent of the participants had low computer video game-playing experience, while 39 percent of the participants classed themselves as having high gameplaying experience.

\subsection{Apparatus}

The participants experienced the IVE in a Trimension ReaCTor system, which is similar to the CAVE system [36]. The Trimension ReaCTor is an immersive projection technology display consisting of three $3 \times 2.2-\mathrm{m}$ walls and a $3 \times 3-\mathrm{m}$ floor. The resolution per screen is 1,024 by 768 , while the brightness is about 300 lumens. The participants wore CrystalEyes stereo glasses and were tracked by an Intersense IS900 system. The system allows the participant to move fully immersed in the environment displayed while viewing it in the correct perspective. Data gathered from the tracker allowed us to record the participants' spatial behavior in the virtual environment. They held a wireless navigation device with an analog joystick. The joystick was used to move around the virtual environment. Participants could also move physically within the confines of the ReaCTor in order to fine tune their position and orientation in relation to the virtual characters. Participants were fitted with Thought Technologies Ltd. ProComp+ electrocardiogram (ECG) sensors on their torso, galvanic skin response (GSR) sensors on their nondominant hand, and a respiration sensor around their torso. These sensors were used to record physiological responses during the experiment. 


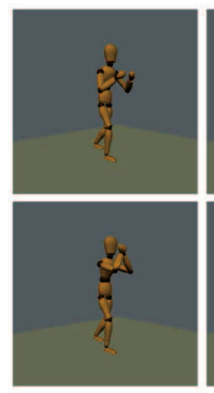

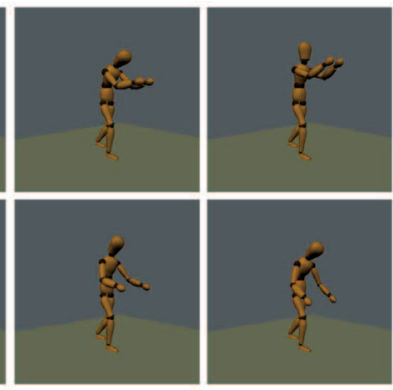

(a)
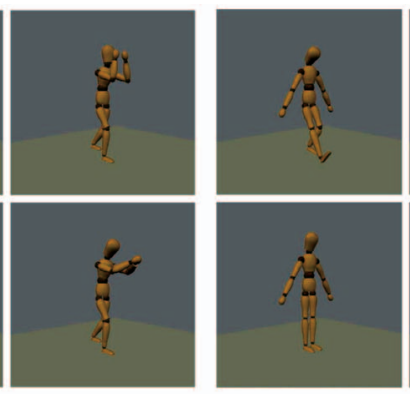
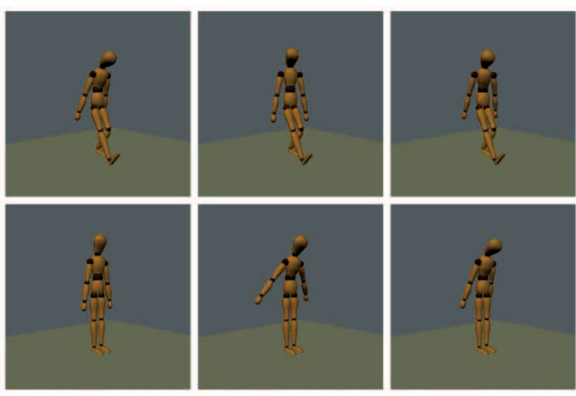

(b)

Fig. 2. Sample of the affective postures adapted from Coulson [4] for the active virtual character. (a) Angry postures. (b) Sad postures.

\subsection{Software}

The software used was implemented on a derivative of Distributed Interactive Virtual Environment (DIVE) $3.3 \times$ which was ported to support spatially immersive systems [37]. DIVE is an Internet-based multiuser VR system in which participants can navigate in a shared 3D space. A VRPN plugin [38] to DIVE was used to enable the logging of tracking data and events. The behavior of the characters was controlled using Tcl scripts which called procedures in Platform Independent Architecture for Virtual Characters and Avatars (PIAVCA) [39]. The PIAVCA is a library for controlling virtual characters through the use of motion data. In addition, a posture loading module enabled the experimenter to assign a set of emotion-grouped postures to each character in the scenario.

\subsection{Virtual Environment: The Maze}

The environment used as a backdrop to the experiment consisted of a training room connected to a centralized virtual maze. The training environment was used to acclimatize participants to viewing and navigating in a virtual environment. The maze was modeled around a central room where participants met the two virtual characters. The central room consisted of eight doors, three of which were connected to three rooms by long interconnecting corridors; a bedroom, a dining room, and a library.

\subsection{The Virtual Characters}

\subsubsection{Visual Appearance}

Two male virtual characters of the same height $(1.7 \mathrm{~m})$ were used in the experiment (Fig. 1). It has been found in previous studies that participants perceive characters to have roles of leadership purely on the basis of their visual appearance or enhanced capabilities [14]. For this reason, a deliberate decision was made to use the virtual character in formal suit to be the active one.

\subsubsection{Postural Animation}

Depending on the condition, the active character was assigned a base set of 32 angry, sad, or neutral postures corresponding to the experiment condition. Fig. 2 shows a subset of the angry and sad postures adapted from Coulson's work [4]. The subset represents the variation of the complete posture set. Coulson's set of postures was designed using a set of hand-defined parameters: Head bend, Chest bend, Abdomen twist, Shoulder swing, Shoulder adduct, Elbow bend, and Weight transfer. Anger was depicted by a backward head bend, an absence of a backward chest bend, no abdominal twist, and arms raised forward and upward. Sadness was characterized by a forward head bend, in addition to a forward chest bend, no twisting, and arms at the side of the trunk. None of the postures in our neutral set overlapped with either the angry or the sad sets. The neutral set comprised of "casual" postures which were a combination of poses which depict head tilts and weight shifts. The neutral set was used in the animation of the active character in the conditions in which neutral postures were needed. The passive character was always assigned a set of 32 neutral postures only.

The posture loading module used a base posture and the set of assigned postures to create a much larger interpolated set of postures [40]. Each new posture in the interpolated set was then produced by assigning a random weight from a uniform distribution to some or all of the basic postures and summing the resulting poses. Each interpolated posture was in keeping with the parameters described by Coulson [4]. The resulting postures were smoothly blended into an animation at intervals generated from an exponential distribution with a mean of 1 second. Each postural shift was then accompanied with the condition-appropriate facial expression. Additional conversation feedback-type animations were created and assigned to the each virtual character. These feedback animations were only used during face-to-face participant-character interactions.

\subsubsection{Facial Expressions}

The PIAVCA facial animation functionality was used to create gaze behaviors, blinking and facial expressions for both virtual characters. A base set of angry or sad facial expressions were used to create the condition-dependent emotional facial expression for the active character (Fig. 3) [41]. Random weights were generated and used at run time to interpolate between the facial expressions resulting in affective facial animation. In previous studies, a more realistic gaze model [7] coupled with semiphotorealistic characters, similar to those used in this experiment, significantly improved the user's perceived quality of communication [42]. Therefore, the same gaze behavior model was implemented in addition to other conversational feedback-type behaviors such as the raising the eyebrows while making a query. Finally, a model to generate blinking 


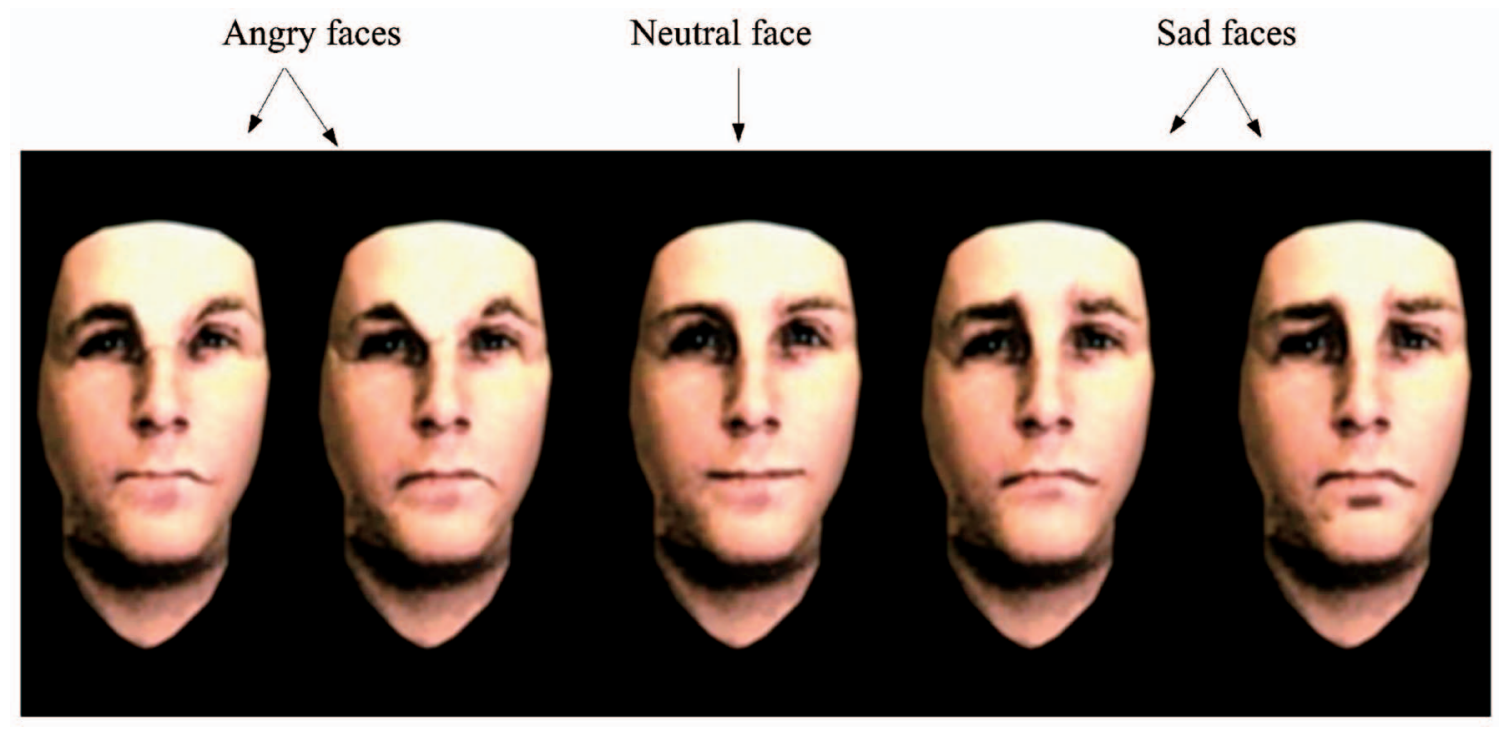

Fig. 3. Sample of the facial expressions created for the active virtual character.

behavior and lip-synching animations was implemented using appropriate facial mesh deformations.

\subsubsection{Behavior Generation}

There were two sets of behaviors assigned to the virtual characters. The first set was used to control the conditiondependent emotional state of the active character toward the passive character, which in turn displayed only neutral behavioral cues. This set of behaviors was only generated when the participant was purely observing the virtual characters in discussion. Once the participant invaded the social space of the virtual characters, the active character glanced at the participant and turned toward the passive character once more as if to stop the discussion. At this point, the first set of behaviors was brought to an end and the participant became the focus of both virtual characters.

The second set of behaviors was used to enable an experimenter-controlled participant-character verbal interaction. In keeping with the active-passive identities created as part of the scenario, only the active character engaged in conversation with the participant. The passive character remained a listener. For instance, when the participant asked a yes/no question, the active character replied vocally and nonverbally by shaking its head while saying "no" or nodding its head while saying "yes". The vocal responses were also accompanied with lip-synching animations. The passive character would do similar but more subtle animations as if to reaffirm the active character's response. Forty-two prerecorded questions and answers were triggered by an experimenter at appropriate times. The range of topics needed for these prerecorded messages was minimal due to the task given to the participants.

\subsection{Task}

Each participant was given the task of counting trash bins after finding three rooms to explore in a virtual maze. They were informed that the virtual characters in the central room of the maze would be able to guide them to three different rooms one after the other. The participants were asked to come back into the central room after exploring each room to get further instructions from the virtual characters.

\subsection{Procedure}

The study was approved by the UCL Ethics Committee for Non-Clinical Research. Two experimenters guided each participant through the experiment. On arrival, the participants were given information, asked to sign a consent form, and complete a number of pre-experiment questionnaires. The participant was then invited to step into the CAVE-like system and fitted with the tracking devices, stereo glasses, physiology monitoring devices, and a microphone. After collecting a baseline for the physiological measures, the participant was given some training in navigating virtual environments. Once the participant was comfortable, he was given the task and asked to complete his task in the virtual maze. The setup and training period took about 10 minutes.

All participants were directed by the virtual characters to three rooms designed to mimic a bedroom, a dining room, and a library. Therefore, each participant interacted with the characters three times. During each interaction, the participant initially saw the virtual characters having a discussion until the participant got close enough to interrupt the characters and get instructions to the next room. During the interaction, an experimenter triggered the virtual characters to ask participants about their experience in the previous room they had visited.

The participant was videotaped throughout his time in the maze while an experimenter noted down any interesting observations on the participants' behavior with the virtual characters. Finally, a number of subjective responses were collected using postexperiential questionnaires and a semistructured debrief interview.

\subsection{Response Variables}

Our key response variable is the extent to which the participants respond to the characters and the virtual sensory data as if it were real sensory data. We measured 
participant response at three levels using several instruments: subjective, physiological, and behavioral.

\subsubsection{Subjective Responses}

The subjective responses collected through questionnaires can be categorized into three indicators: "being there," operational presence, and operational copresence. The first indicator was based on the SUS questionnaire and measured the extent to which participants report a sense of "being there" in the environment. [43]. For instance, one of the five questions in the SUS questionnaire was "During the time of the experience, which was strongest on the whole, your sense of being in the maze, or of being in the real world of the laboratory?"

The next two indicators were designed based on the concept of operational presence defined in [9]. In this approach, presence is taken as the extent to which participants act and respond to virtual sense data as if it were real, where "response" is considered at different levels ranging from physiological through to cognitive. Similar to the SUS, the operational presence questionnaire is based on the types of statements that people have made in postexperimental interviews over many years. The following six questions were used to construct the second indicator (operational presence):

- How much did you behave within the maze as if the situation were real?

- How often did you find yourself automatically behaving within the maze as if it were a real place?

- How much was your emotional response in the maze the same as if it had been real?

- How much were the thoughts you had within the maze the same as if the maze had been a real situation?

- How much were you thinking things like "I know this isn't real" but then surprisingly finding yourself behaving as if it was real?

- To what extent were your physical responses within the maze (e.g., heart rate, blushing, sweating, etc.) the same as if the maze had been a real situation?

The third indicator (operational copresence) also consisted of six questions and is similar to the operational presence indicator except that it assessed the extent to which participants reported behaving and responding as if the virtual characters were real. All these three indicators were scored on a 7-point Likert-type scale where 1 corresponded to strong disagreement and 7 was strong agreement. A score of 6 or 7 was counted as a high reported presence response.

An additional questionnaire was used to ascertain the participants' post hoc evaluation of each characters' underlying emotional state toward the other character. Participants were asked to choose a label that best represented the characters' emotional state out of seven possible options: surprised, afraid, angry, happy, disgusted, sad, and neutral.

In addition to questionnaires, semistructured interviews were conducted in order to ascertain the participants' impressions of the virtual characters. The interviews were audio-taped and transcribed verbatim before analysis. This offered the advantage of allowing the experimenter to conduct informal, conversational-like in-depth interviews with participants while adhering to some level of structure. A general framework of open-ended evaluative questions with room for follow-up questions was planned beforehand and followed through in no particular order during the interviews.

At the beginning of the interview, the participant was asked to describe his experience in the maze from start to finish. This allowed the participant to recollect and describe their experience in their own words. It also allowed the experimenter to note down specific parts of the particular participant's experience that could be explored further to uncover interesting findings. This was then followed by questions focused on the participant's impression of the virtual characters. There were four broad areas covered in the interviews: the participant's general impression of what was going on in the virtual maze, the participant's feeling of presence and responses, the extent to which participant had an uninterrupted experience, and the participant's impression of the virtual characters.

\subsubsection{Physiological Responses}

The participants' GSRs, ECG, and respiration were monitored and recorded using the Procomp+ device.

\subsubsection{Behavioral Responses}

One of the ways in which the body can be used to express internal states is through the manner in which an individual stands relative to other people. This spatial use of the body is called proxemics [6]. Bailenson et al. [31] argued for proximal responses to be utilized as a behavioral measure of presence. The advantage of using proximal responses as a gauge is that tracking data of participants in the system is cheaply available.

\subsection{Explanatory Variables}

A number of demographic variables were recorded including: the participants' age (Age), their computer literacy (Literate), experience in programming (Program), level of experience with VR systems (VR), their level of computer game playing experience (Game), and amount of time spent playing video games per week (Gametime). In addition, there were four standard psychological questionnaires used:

- Social Avoidance and Distress (SAD). 28 questions assessing social anxiety in everyday life [44].

- State Trait Anxiety Inventory (STAI). 40-item questionnaire assessing two measures of anxiety: state and trait [45].

- Affective Perception Test (APT). 14-item questionnaire gauging a person's level of emotional perception [46].

- Emotion Contagion (EC). 15-item scale measure of susceptibility to catching the emotions of others [47].

\subsection{Method of Analysis}

\subsubsection{Subjective Questionnaire Data}

Since Likert scales are ordinal, it is not suitable to treat the responses as if they were on an interval scale. Therefore, the same logistic regression method used in [42] was applied to our questionnaire responses. In this method, the response 
variables are thought of as counts of "successes" in a number of trials corresponding to the number of questions. All scores of " 6 " or " 7 " on the 7-point scale are counted as a high response. Under the null hypothesis of no relationship between the explanatory and response variables, the responses would have a binomial distribution as required in binomial logistic regression [48]. This is equivalent to an analysis of covariance but using the binomial distribution and logistic link function rather than the Normal distribution with identity link.

In this regression model, the deviance is the appropriate goodness-of-fit measure and has an approximate Chisquared distribution with degrees of freedom depending on the number of fitted parameters. A rule-of-thumb is that if the deviance is less than twice the degrees of freedom then the model overall is a good fit to the data (at the 5 percent significance level). More importantly, the change in deviance as variables are deleted from or added to a fitted model is especially useful, since this indicates the significance of that variable in the model. A large change of deviance relative to the Chi-squared distribution indicates a significant contribution of the variable to the overall fit of the regression model. Analysis of the data was conducted using GLIM [49].

\subsubsection{Subjective Interview Data}

The method of analysis used for the interview responses was a form of thematic analysis [50]. The analyst defined an initial set of categories. This was done by reading through all the text before the coding process and identifying a set of broad categories which could be used to structure the data. This process also allows the analyst to note down particularly interesting observations made by the participant.

Once the initial set of categories are defined, a detailed analysis is conducted by going through the data, categorizing it, and labeling it with a code. ATLAS.ti [51] was used during the coding phase of the qualitative analysis. The number of instances within the text that fitted into each category was then summarized to locate themes that related to the research questions. Since there were a set of research questions associated with the experiment, a problem-driven view of the analysis was conducted. Within this approach, the analyst started from the research question and then looked for analytical paths from the sample data to the answers. ATLAS.ti allows the analyst to use links between codes/categories to help define the possible relationship between categories. This facility was then used to interpret the themes and possible cause-and-effect relationships between them.

\subsubsection{Physiological Responses}

The skin conductance responses (SCR) extracted from the GSR recordings were the focus of physiological analysis. Skin conductance takes effect between about 2 and 10 seconds after the stimuli [52]. The primary analysis compared and contrasted the peaks in GSR recordings experienced between conditions. Analysis was conducted on the participants' GSR recording by using the number of SCRs as the main variable. Since there is no standard for defining an SCR, throughout this paper, an SCR is defined
TABLE 2

Summary of the Overall Model Fitted for the "Being There" Response Variable in the Angry Conditions

\begin{tabular}{|l||c|c|c|c|}
\hline & $\chi^{2}$ & d.f. & Association & $\sim$ P value \\
\hline $\begin{array}{l}\text { Neutral Posture } \\
\text { Neutral Face }\end{array}$ & 6.9700 & 1 & + & 0.0082 \\
\hline Age & 25.01 & 1 & + & $<0.0001$ \\
\hline Gametime & 12.95 & 1 & + & 0.0003 \\
\hline Literate & 7.23 & 1 & + & 0.0072 \\
\hline Program & 12.22 & 1 & + & 0.0004 \\
\hline VR & 14.25 & 1 & - & 0.0002 \\
\hline SAD & 7.43 & 1 & - & 0.0064 \\
\hline
\end{tabular}

Deviance $\left(\chi^{2}\right)$ of 51.30 on 18 d.f.

as a peak rise to a maxima in the GSR of at least 0.1 microsiemens over 5 seconds [33]. The number of SCRs during a period of interest was analyzed to uncover factorrelated differences by fitting the explanatory variables to a log-linear regression model with a Poisson distribution, since under the null hypothesis of no relationship between this response variable and the explanatory and/or independent variables, the number of SCRs per unit time would follow a Poisson distribution. Additionally, the number of SCRs experienced by the participant depends on their particular physiology. Some people have a high rate of spontaneous SCRs (labiles) and others a much lower rate (stabiles). In order to take into account the physiology of the participant, the rate of SCRs recorded during baseline resting periods of the experiment was always fitted into the regression model. Similarly, the duration of the experience was also included in the model to eliminate the effect of having an increased number of SCRs due to the amount of time spent in the experience.

\subsubsection{Objective Proximal Responses}

Data corresponding to the periods of interest was extracted from the participant position recordings. A visual inspection of the data revealed that during interaction with the virtual characters, the participants stood facing the characters and made relatively small movements. A set of variables including the minimum and average interpersonal distances, maintained by the participants during their interactions with the characters, were computed and analyzed using ANOVAs.

\section{RESULtS}

\subsection{Findings from the Questionnaire Responses}

There were significant factor-related results in the responses collected through the SUS ("being there") and "operational copresence" indicators in the Angry conditions. In the Sad conditions, the "operational presence" and "operational copresence" indicators yielded significant results. These results have been presented in Tables 2, 3, 4, and 5. The explanatory variables included in the final regression model are also observable in the tables and are listed in the order of the significance of their contribution to the overall model. The $\sim \mathrm{P}$ values reported in the tables correspond to deleting the variable from the fitted model. It indicates the significance of contribution of the corresponding variable, 
TABLE 3

Summary of the Overall Model Fitted for the "Operational Presence" Response Variable in the Sad Conditions

\begin{tabular}{|l||c|c|c|c|}
\hline & $\chi^{2}$ & d.f. & Association & $\sim$ P value \\
\hline Neutral Posture & 7.36 & 1 & + & 0.0067 \\
\hline Literate & 9.41 & 1 & + & 0.0022 \\
\hline EC & 6.63 & 1 & - & 0.0100 \\
\hline Game & 11.23 & 1 & - & 0.0008 \\
\hline
\end{tabular}

Deviance $\left(\chi^{2}\right)$ of 49.40 on 23 d.f.

i.e., if the variable was deleted from the model, the $\sim \mathrm{P}$ value indicates how worse the fit would be.

\subsubsection{Presence Response-SUS "Being There"}

In the Angry conditions, an interaction effect was observed between the two factors: type of facial cue and type of postural cue. Separately, the neutral postures and the neutral facial expressions were associated with less reported presence as measured using the "being there" indicator. However, the neutral posture with the neutral facial expression was associated with higher reported presence (Table 2).

The overall model fitted had a deviance $\left(\chi^{2}\right)$ of 51.30 on 18 d.f. At the 5 percent level of significance, $\chi^{2}$ has to be less than 28.87 at 18 d.f. for a model with good fit. Therefore, the deviance of our model indicates that although the individual terms are significant, the overall explanatory power of the model is low. This means that there are important explanatory variables missing. Similar analysis on the "being there" presence response variable for the Sad condition revealed no significant factor-related results.

\subsubsection{Presence Response-Operational}

Analysis of the operational presence response variable within the Angry conditions did not yield any significant factor-related results. However, in the Sad condition, the neutral postures was associated with higher reported presence. Table 3 shows the results including the explanatory variables fitted to the regression model.

The overall model fitted for the operational presence response variable had a deviance of $\chi^{2}=49.40$ on 23 d.f. At the 5 percent level of significance, $\chi^{2}$ has to be less than 35.17 at 23 d.f. for a model with good fit; therefore, the overall explanatory power of this model was low as well.

\subsubsection{Copresence Response-Operational}

The operational copresence indicator was designed to capture the responses of the participants to the virtual characters. Tables 4 and 5 depict the significant variables fitted into the overall model, deviance $\left(\chi^{2}\right), \sim \mathrm{P}$ value, and direction of association.

TABLE 4

Summary of the Overall Model Fitted for the "Operational Copresence" Response Variable in the Angry Conditions

\begin{tabular}{|l||c|c|c|c|}
\hline & $\chi^{2}$ & d.f. & Association & $\sim$ P value \\
\hline Neutral Posture & 4.67 & 1 & + & 0.0304 \\
\hline Trait & 8.86 & 1 & - & 0.0029 \\
\hline
\end{tabular}

Deviance $\left(\chi^{2}\right)$ of 66.30 on 25 d.f.
TABLE 5

Summary of the Overall Model Fitted for the "Operational Copresence" Response Variable in the Sad Conditions

\begin{tabular}{|l||c|c|c|c|}
\hline & $\chi^{2}$ & d.f. & Association & $\sim$ P value \\
\hline Neutral Posture & 8.79 & 1 & + & 0.0030 \\
\hline Neutral Face & 5.73 & 1 & + & 0.0167 \\
\hline Game & 5.05 & 1 & - & 0.0246 \\
\hline Gametime & 5.91 & 1 & + & 0.0150 \\
\hline VR & 7.33 & 1 & + & 0.0068 \\
\hline
\end{tabular}

Deviance $\left(\chi^{2}\right)$ of 26.09 on 22 d.f.

In both the Angry and the Sad conditions, the neutral postures were associated with an increase in reported copresence. The overall model for the Angry conditions included Posture and the participant's level of trait anxiety ( $\chi^{2}=66.30$ on 25 d.f.). At the 5 percent level of significance, $\chi^{2}$ has to be less than 37.65 at 25 d.f. for a model with good fit; therefore, the overall explanatory power of this model was low. In the Sad condition, as well as the postural cues, the neutral facial cues were also associated with higher reported copresence. The overall model has a deviance of $\chi^{2}=26.09$ on 22 d.f. Unlike the Angry conditions, the overall model for the Sad conditions is of a good fit since $\chi^{2}$ has to be less than 33.92 at 22 d.f. at the 5 percent level of significance.

\subsubsection{Accuracy in Recognizing Underlying Emotion}

After the experiment, participants were given a list of seven possible emotional states (Section 4.11.1) and asked to make a judgement of the virtual characters' underlying emotional state toward each other. Tables 6 and 7 show the results in the conditions with one or more affective behavioral cues.

In the Angry conditions, all seven participants in the conditions with emotional facial expressions and emotional postures accurately judged correctly that the active character's emotional state toward the passive character was angry. Five participants in the condition with affective facial cues only judged the active character's emotional state as angry. Four participants in the condition with affective postural cues only accurately judged the active character as being angry toward the passive character while two participants thought the active character was disgusted at

TABLE 6

Angry Conditions: Each Participant's Post Hoc Judgment of Each Characters' Emotional State toward the Other

\begin{tabular}{|c|c|c|c|c|c|c|}
\hline & \multicolumn{2}{|c|}{$\begin{array}{l}\text { Emotional } \\
\text { face } \\
\text { Emotional } \\
\text { posture }\end{array}$} & \multicolumn{2}{|c|}{$\begin{array}{l}\text { Emotional } \\
\text { face } \\
\text { Neutral } \\
\text { posture }\end{array}$} & \multicolumn{2}{|c|}{$\begin{array}{l}\text { Neutral face } \\
\text { - Emotional } \\
\text { posture }\end{array}$} \\
\hline & Active & Passive & Active & Passive & Active & Passive \\
\hline $\mathbf{1}$ & Angry & Neutral & Angry & Afraid & Angry & Afraid \\
\hline 2 & Angry & Afraid & Angry & Afraid & Disgust & Surprise \\
\hline 3 & Angry & Sad & Angry & Afraid & Angry & Afraid \\
\hline 4 & Angry & Afraid & Angry & Neutral & Disgust & Afraid \\
\hline 5 & Angry & Afraid & Angry & Neutral & Neutral & Neutral \\
\hline 6 & Angry & Neutral & Neutral & Angry & Angry & Surprise \\
\hline 7 & Angry & Afraid & Neutral & Neutral & Angry & Neutral \\
\hline
\end{tabular}

The active character was designed to portray anger, while the passive character was designed to be "neutral." 
TABLE 7

Sad Conditions: Each Participant's Post Hoc Judgment of Each Characters' Emotional State toward the Other

\begin{tabular}{|l|l|l|l|l|l|l|}
\cline { 2 - 7 } \multicolumn{1}{c|}{} & \multicolumn{2}{|l|}{$\begin{array}{l}\text { Emotional } \\
\text { face } \\
\text { Emotional } \\
\text { posture }\end{array}$} & \multicolumn{2}{l|}{$\begin{array}{l}\text { Emotional } \\
\text { face } \\
\text { Neutral } \\
\text { posture }\end{array}$} & \multicolumn{2}{l|}{$\begin{array}{l}\text { Neutral face } \\
\text { Emotional } \\
\text { posture }\end{array}$} \\
\cline { 2 - 7 } & Active & Passive & Active & Passive & Active & Passive \\
\hline $\mathbf{1}$ & Angry & Neutral & Angry & Afraid & Angry & Neutral \\
\hline $\mathbf{2}$ & Disgust & Sad & Angry & Neutral & Neutral & Angry \\
\hline $\mathbf{3}$ & Neutral & Surprise & Happy & Neutral & Neutral & Neutral \\
\hline $\mathbf{4}$ & Neutral & Neutral & Angry & Afraid & Neutral & Neutral \\
\hline $\mathbf{5}$ & Neutral & Neutral & Surprise & Happy & Disgust & Afraid \\
\hline $\mathbf{6}$ & Angry & Neutral & Neutral & Angry & Neutral & Afraid \\
\hline $\mathbf{7}$ & Neutral & Neutral & Angry & Neutral & Angry & Afraid \\
\hline
\end{tabular}

The active character was designed to portray sadness, while the passive character was designed to be "neutral."

the passive character and one participant thought the active character was neutral.

In the Sad conditions, not even a single participant in the conditions with affective behavioral cues (facial or postural) identified the active character's emotional state toward the passive as sad. This was the case even in the conditions where the active character displayed congruent and affective behavioral cues. In the control (Neutral) condition, two participants thought that active character was happy, while two thought the active character was being angry. The last participant in the Neutral condition judged the active character as being sad.

The passive character was designed to always portray the same behavioral cues throughout the whole experiment; however, only 20 out of the 49 participants perceived the passive character to be neutral. It appears that the participants perceive the affective state of the passive character as a function of the active character's behavioral animations.

\subsection{Findings from the Skin Conductance Responses}

The number of SCRs was calculated for the whole period in which participants observed and interacted with the virtual characters. There were three sets of SCR data for each participant corresponding to the three times the participant interacted with the character. Therefore, there were three factors to take into account in the linear regression analysis: the type of postural cue, the type of facial expression, and the number of times the participant had met the characters previously (Visit). The log-linear model included the rate of SCRs recorded during the baseline period of the experiment and the duration of the experience in order to eliminate effects due to those factors.

In the Angry conditions, both neutral postures and neutral facial expressions were positively associated with the number of SCRs (Table 8). The overall model fitted had a deviance $\left(\chi^{2}\right)$ of 146.40 on 47 d.f. At the 5 percent level of significance, $\chi^{2}$ has to be less than 64.00 at 47 d.f. for a model with good fit; therefore, the overall explanatory power of this model was low.

In the Sad conditions, the number of times (Visit) the participants had interacted with the characters previously
TABLE 8

Summary of the Overall Model Fitted for the Number of SCRs in the Angry Conditions

\begin{tabular}{|l||c|c|c|c|}
\hline & $\chi^{2}$ & d.f. & Association & $\sim$ P value \\
\hline Neutral Posture & 10.63 & 1 & + & 0.0011 \\
\hline Neutral Face & 9.25 & 1 & + & 0.0023 \\
\hline APT & 20.39 & 1 & - & $<0.0001$ \\
\hline VR & 19.37 & 1 & - & $<0.0001$ \\
\hline Age & 40.33 & 1 & - & $<0.0001$ \\
\hline Literate & 20.46 & 1 & + & $<0.0001$ \\
\hline Gametime & 4.63 & 1 & - & 0.0315 \\
\hline
\end{tabular}

Deviance $\left(\chi^{2}\right)$ of 146.40 on 47 d.f.

was a significant factor in the fitted model. The second period during which the participants approach and interact with the characters is positively associated with the number of SCRs while the third period is negatively associated with the number of SCRs. Like the Angry conditions, neutral facial expressions were positively associated with the number of SCRs; however, unlike the Angry conditions, neutral postural cues were negatively associated with the number of SCRs (Table 9). The overall model had a deviance $\left(\chi^{2}\right)$ of 79.90 on 46 d.f. At the 5 percent level of significance, $\chi^{2}$ has to be less than 62.83 at 46 d.f. for a model with good fit; therefore, the overall explanatory power of this model was low as well.

\subsection{Findings from the Proximal Responses}

Without exception, all participants started the participantcharacter interaction while they were stationed in front of the characters. Most participants continued to stand in the same spot throughout the interaction as well. Independent of condition, during the first interaction, 44 out of the 49 participants maintained a smaller interpersonal distance with the active character in comparison with the passive character. The number of participants who maintained a smaller interpersonal distance with the active character reduced to 41 during the second interaction and to 31 in the final interaction. However, an ANOVA between conditions did not reveal any significant differences (Table 10).

\subsection{Findings from the Interviews}

Two out of 49 participant interviews were excluded from the analysis since the recordings of the interviews were of poor audio quality. Eleven participants reported that their

TABLE 9

Summary of the Overall Model Fitted for the Number of SCRs in the Sad Conditions

\begin{tabular}{|l||c|c|c|c|}
\hline & $\chi^{2}$ & d.f. & Association & $\sim$ P value \\
\hline Neutral Posture & 3.95 & 1 & - & 0.0468 \\
\hline Neutral Face & 7.59 & 1 & + & 0.0059 \\
\hline Visit & 7.59 & 2 & & 0.0044 \\
\hline Visit(2) & & & + & \\
\hline Visit(3) & & & - & \\
\hline EC & 27.05 & 1 & - & $<0.0001$ \\
\hline VR & 10.60 & 1 & - & 0.0011 \\
\hline Age & 4.91 & 1 & - & 0.0267 \\
\hline Gametime & 5.03 & 1 & + & 0.0250 \\
\hline
\end{tabular}

Deviance $\left(\chi^{2}\right)$ of 79.90 on 46 d.f. 
TABLE 10

Means and Standard Deviations of Average and Minimum Interpersonal Distances Maintained by the Participants with the Active and Passive Characters

\begin{tabular}{|l|l|c|c|}
\hline \multicolumn{2}{|c|}{} & \multicolumn{2}{|c|}{ Interpersonal Distances } \\
\cline { 3 - 4 } \multicolumn{2}{|c|}{} & Active & Passive \\
\hline \multirow{2}{*}{ First Interaction } & Average & $2.8 \pm 0.6$ & $3.1 \pm 0.6$ \\
\cline { 2 - 4 } & Minimum & $1.3 \pm 0.8$ & $1.8 \pm 0.6$ \\
\hline \multirow{2}{*}{ Second Interaction } & Average & $2.9 \pm 3.0$ & $3.1 \pm 3.0$ \\
\cline { 2 - 4 } & Minimum & $1.4 \pm 0.5$ & $1.8 \pm 0.6$ \\
\hline \multirow{2}{*}{ Third Interaction } & Average & $3.5 \pm 0.6$ & $3.5 \pm 0.6$ \\
\cline { 2 - 4 } & Minimum & $1.6 \pm 0.8$ & $1.7 \pm 0.7$ \\
\hline
\end{tabular}

own responses in the virtual maze were not realistic, while 23 participants reported that their responses and behavior in the maze were realistic. Thirteen participants reported experiencing a mixture of both realistic and unrealistic responses during the experiment. During the analysis of the semistructured interviews, it became clear that the interpretation of the behaviors of the virtual characters in the maze depended heavily on the participant. For instance, one participant reported that the active virtual character seemed to be surprised by the detailed answer the participant had given during the interactions in the experiment: "I was asked to say what was in the room, the expression [reaction] to what I had said was different to the first time and the guy looked a bit surprised (P7112)." This comment is especially interesting as the virtual characters were designed to display no affective cues during the periods of the experiment where there was face-to-face interaction with the participants-i.e., when the participant was talking to them.

The analysis focused on the variety of realistic responses and interpretations of the scenario reported by all the participants. The findings were in keeping with the premise that a visual likeness to the human form is not necessarily the primary factor responsible in evoking appropriate participant responses to virtual humans. Out of the 36 participants who reported experiencing realistic responses, two participants attributed their responses to the humanoid form of the virtual characters that is the geometry and textures that make up the visual properties of the virtual character. However, most participants (34) reported that their responses were elicited through the behavior (animation) of the virtual characters: "I was thinking thank God, this is not a real environment because you know, the way they were going at each other, I would probably rather not approach them at the moment. So that seemed quite - how do you say? — realistic (P3121)."

\subsubsection{Impressions of the Interaction between the Virtual Characters}

Regardless of experimental condition, participants reported that their judgement of the interaction between the active and passive virtual characters were made based on two cues: body language of the virtual characters and the audio properties of the verbal interaction. Only two participants specifically reported using facial expression as a cue to the virtual characters' underlying emotional state. One of the two participants reported that the passive virtual character's facial expression was key to guiding his impressions of the virtual characters even though there were no affective behavioral cues specifically designed for the passive character.

Previous studies suggest that individuals, in the physical world, use different modalities of expression in a combined and highly synchronized fashion in portraying an emotional state [13]. Similarly, the participants in this study reported using the overall behavior of the virtual characters or body language of both virtual characters in deducing the emotional states of the virtual characters. Out of 47 participants, 16 participants reported using the virtual characters' postural cues (body language). Five participants reported relying on the virtual characters' overall behavior to judge their emotional state. Eleven participants reported paying attention to vocal cues while 10 participants reported reliance on body language, overall behavior, and vocal cues to judge the virtual characters' emotional states. The remaining participants were either more interested in exploring the virtual maze and did not remember much about the virtual characters (3) or viewed the virtual characters solely as objects and were unable to assign a psychological state to them (2).

Generally, participants reported the interaction between the virtual characters as either a conversation or an argument. In either case, participants interpreted the behavior of the virtual characters in keeping with their perceived context. Nine participants reported that the virtual characters looked engrossed in their conversation since the virtual characters only responded after the participants were within $3.25 \mathrm{~m}$. This impression was associated with the virtual characters' overall focus of behavior (directed body language) toward each other combined with the distance at which the virtual characters were triggered to animate responses toward the participants: "They seem to be busy with themselves because they didn't even like look at me or anything when I walked into that room. They obviously didn't notice that I was there until I was literally right in front of them. (P3121)". Out of the 15 participants who reported that the virtual characters were involved in an argument, 11 were in the condition where the active character displayed angry postural cues toward the passive character and 2 were in the conditions where the active character displayed only angry facial cues toward the passive virtual character. These 11 participants relied on body language as the main cue for their interpretation. However, their assessment was based on the body language of both virtual characters: "His [active] body language was a bit aggressive. Not with me but with the other guy [passive]. You can see him [active] bending and shaking his hands, his arms actually and he was bending, he must have been desperate. The other guy [passive] was just assuming all the blame, just nodding his head. Because you don't really know what they were talking or arguing about but the attitude was one [passive] of submission and the other one [active] of aggression. (P2111r)". Other terms participants used to describe the interaction between the virtual characters were talking (20), a heated one-sided conversation where the active virtual character was verbally chastising or disagreeing with the passive virtual character (10) and complaining to the passive virtual character (2). Throughout the study, participants consistently reported 
using cues portrayed by both virtual characters to form impressions of the virtual scenario and to evaluate the underlying emotional state of each virtual character toward the other. In addition to the virtual character's perceived emotional state, the body language of the virtual characters was associated with the virtual character's perceived awareness of the participant and the active virtual character's perceived higher status (see Section 5.4.5).

\subsubsection{Awareness and Reactive Behavior}

An issue dealt with in the postexperiment questionnaire, partially to check if the virtual character behaviors were portrayed appropriately, was the degree of responsiveness of the virtual characters to the participants. Participants were asked to indicate if they thought each character was 1) responsive to them, displayed the right social cues and portrayed perceived awareness and 2) interacted with them, displayed the right conversational behaviors and gave the right verbal responses. All the participants felt that the active virtual character was responsive and interacted with them. Interestingly, a few participants felt that the passive character was responsive and even more surprisingly three participants felt that the passive character was interactive.

Participants reported that the virtual characters were aware of their presence and responded appropriately using a variety of nonverbal behaviors. Forty-four participants reported that they felt that the virtual characters were aware of them. The participants reported that they got this impression because the virtual characters noticed the participant (8), acknowledged the participant's presence (5), halted the conversation that was already going on between the virtual characters (21), turned toward the participants (41), and maintained eye contact (33). In this case, perceived awareness is directly linked to the virtual character's ability to direct responsive behavioral cues toward the participant. One of the most significant behavioral cues that participants reported as being characteristic of perceived awareness was gaze (eye contact). This included the process of redirecting gaze behavior from the other virtual character to the participant, i.e., halting the ongoing conversation, turning toward the participant, and responding to the participant's queries while maintaining eye contact: "Purely by when you walked to them and they turned around, both facing you and in a way, almost introduce themselves or ask "how can we help". That definitely was a sense that "ah, ok I am now in the presence of these two people". (P7112)". Fourteen participants also reported that the virtual characters had a sense of awareness when the virtual characters appeared to have some knowledge pertaining to either the spatial properties of the virtual maze or the participant's activity in the virtual maze: "I would say especially after the third time, when I was looking for the door and I was told to turn behind me, so I assume that they must have been aware of my presence because otherwise it would be difficult for them to know. (P5211)".

This effect was especially pronounced in the case of the active character. Participants were more certain about the perceived awareness of the active virtual character either because they paid more attention to the more interactive active virtual character or because the passive virtual character did not engage them in an active interaction. As expected, nearly all the participants paid more attention to the active virtual character due to its perceived enhanced responsiveness in comparison to the passive virtual character.

\subsubsection{Limited Interaction-Due to Characters or Social Context?}

In addition to the visual appearance of the virtual characters, the limited vocal responses and interaction capabilities offered by the virtual characters was cited as the most significant reason for not responding to the characters as if they were real. Thirteen participants were especially attuned to timing errors in the active character's responses due to lag in the system. These participants attributed the timing errors to computer glitches in the software and a lack of complex responses to the participants. However, this was not always the reason for limited interactions.

The level of interaction between the participant and the character depended on the participant's expectations. Thirteen of the forty-seven participants did not expect any queries or complex responses from the virtual characters. A further 12 participants did not attempt to engage the virtual characters in conversation since they felt it would be fruitless or unnecessary: "Because of the task and you don't expect the virtual people to have all the responses available for you, you just think they are going to say go to the room and that is all you think you will get, so I definitely didn't do much more than just ask for directions or something. (P7112)". Since the task involved using the virtual characters as guides to explore the virtual maze, these 12 participants paid very little conscious attention to the virtual characters. However, in cases where the participant did not interact with the characters due to the nature of the task, the participant did not report their behavior or responses to the virtual characters as being unrealistic.

A few participants did not attempt to engage the virtual characters in an interaction initially because they had no expectations of the virtual character's abilities. These participants grew more comfortable with the interactions after they had gauged the virtual character's abilities through experience. When the virtual characters responded in a manner that surpassed the participant's expectations, participants attributed a sense of realism to the virtual characters: "They caught me off guard a couple of times because they asked me what I had seen in the room, and I didn't anticipate them in doing that. So that brought an extra bit of realism to them. (P1211)". Five participants were surprised that they had responses to the virtual characters especially when they had strong prior expectations of the virtual characters' ability: "One thing is exactly like I expected which is like you know graphically they just won't look like real people, and therefore, you will find it hard to take them seriously as real people. But the fact that they were talking and seem a bit negative ... that's a bit weird ... was a real sort of bad vibes going on there. Which you know bad vibes from a bunch of graphics. That was maybe a bit more than I expected. (P3121)". However, four participants expected the virtual characters to be highly informed and have better behavioral capabilities, despite their visual appearance. This impression might have been 
developed after the participant was told that the virtual characters would act as guides to the virtual maze. For instance, one participant expected to conduct a more naturalistic and interactive conversation with the virtual characters: "It was a more like a automatic relation, you know. I ask for information, he provided the information. It was a more descriptive relation, ok, you have to do that, and I was waiting for the information to continue. (P2121)". After unsuccessfully trying to engage the virtual characters in a more complex interaction, these participants resigned to simply getting the information required to complete their task.

Some participants reasoned that the limited interaction was either due to the social context within which the interaction took place, a lack of the virtual character's interest in the participant or the virtual character's previous emotional state. Four participants attributed the uneasy interaction to the state of the virtual characters: "Regarding the character on the left [passive], it's because he seemed completely tied up with his own problem and didn't really turn to me and didn't try to make, try to really communicate with me at all, and with the character on the right [active], I think it's, he seemed dutiful rather than welcoming. More to do in what wasn't said, rather than what was said. I think normally if someone was actually being friendly, they would ask you out of politeness or courtesy, what you thought of the experience or what, lets say what you found out in the rooms. (P4121r)". Six participants attributed the limited interaction to the active virtual character's lack of interest in the participants: "I got more of a response out of the right guy [active], he seemed like he was you know quite confident. They both seemed pretty disinterested. Pretty kind of, you know, focused on themselves. (P6212)". In the case of the passive and less responsive virtual character, three participants attributed the limited levels of interaction with an intimidating persona. Five other participants perceived the characters as not welcoming, impatient, and too engrossed in the previous interaction with each other. Since the active virtual character was more heavily involved during the face-to-face interaction, the active character in particular was perceived as impatient. One participant reported that the characters were trying to cut the interaction short since the virtual characters were interested in continuing their previous conversation with each other. Another participant reported that the virtual characters did not want to have an interaction with him initially but this changed during the experiment. Again, the interpretation of the virtual characters' perceived behavior (even limitations) is interpreted in keeping with the scenario presented to the participant.

\subsubsection{Participant Responses to the Virtual Characters}

One of the most significant responses reported by participants was the feeling of intrusion when interrupting the virtual characters at the end of the approach periods of the experiment. This was especially the case when the virtual characters were perceived to be involved or engrossed in an argument or disagreement with each other. Twelve participants reported feeling hesitant, embarrassed, and uncomfortable upon intruding on the virtual characters' conversation: "I walked up to the 2 people that seemed to have been in an argument. And since I didn't want to interfere with the argument, I just asked them that I had a task and I needed help.
The main thing was that I saw them in a conflict, some type of argument, so I held back interacting with them. Similarly if I saw two people fighting in the real world, I won't approach the person anymore ... I would choose the one that weren't in conflict with each other... I kind of felt bad for the person on the left [passive]. (P7111)". The feeling of intrusion was associated either with the virtual characters' behaviors toward each other before the interaction with the participant or the virtual characters' perceived behavior after the interaction with the participant. In keeping with the feelings of some participants that they were intruding into the conversation between the virtual characters, two participants perceived the characters as being unfriendly and described their behavior as unhelpful and dismissive.

Another response that surprised nine participants was their tendency to be automatically polite toward the virtual characters despite knowing that the virtual characters were not real: "I didn't think they would be able to have a discussion back with me. So I anticipated a very limited capacity because they were generated by the computer, so I just sort of spoke to them "hello, which room am I suppose to go into?". I was aware that I was polite though because I kept saying "thank you" after they told me where to go which obviously is unnecessary because they are not real. (P1211)". Some participants reasoned that this was because the virtual characters were more realistic than they had expected. One participant reported feeling hesitant in carrying out his personal plan to try and listen in on the virtual characters' conversation since it would have been socially impolite: "I was kind of curious to look at them but then I probably moved because it felt socially awkward to just... because you did have the sense that they were responding to you ... It felt like if I just kept standing there and staring at them, it would have been kind of inappropriate. (P6212)". This is particularly interesting since the participant's curiosity to test the virtual characters was overcome by his desire to maintain social norms.

\subsubsection{Participants' Impressions of the Virtual Characters' Persona}

The virtual characters were designed to have a neutral attitude toward the participants; however, the qualitative analysis of the interviews uncovered that participants had varying impressions of the virtual characters' attitude toward them. More than 45 percent of the participants reported that the virtual characters had a formal or neutral attitude toward them with slight variations. In keeping with the social context and the perceived roles of the virtual characters, their behavior was described as polite, slightly upset at being interrupted but still neutral, friendly but formal, sufficiently accommodating, instructional, in keeping with the situation, and dutiful and generally behaved as the participants expected.

Other participants reported different attitudes for each virtual character since the active virtual character was more interactive than the passive virtual character. Even though the only difference between the behavioral cues displayed by the virtual characters directly toward the participants was in the level of energy (physical expression), the overall effect seemed to have created a noticeable difference between the apparent personalities of the virtual characters. 
Participants interpreted the behaviors of the virtual characters in a holistic manner by comparing the active virtual character with the passive virtual character in parallel: "I thought he [active] was kind of a warm person in a computer way and the other person [passive] was very cold. He just didn't actually interact at all. (P5212)". Furthermore, four participants perceived a change in the virtual character's behaviors even though there was none: "I think the first two meetings, they seemed to ... they were quite similar you know, it was kind of a negative atmosphere. But then the third meeting, it seemed to have been resolved. (P1221)".

Thirty-nine out of 47 participants compared the interaction they observed between the virtual characters with similar interactions they had witnessed in real life and assigned roles with higher authority to the active virtual character due to a combination of the perceived persona of the virtual characters: "He [active] was obviously the one dominating between the two of them and he looked more, I suppose authoritative because he had the jacket and [looked] smart (P5111)"; and the attire of both virtual characters: "The kind of impression I got was of the guy in the suit [active] being the boss of the guy in the white t-shirt [passive] basically. He worked for him or something. Maybe that was to do with the suit. (P7221)".

Participants also assigned a position of higher authority to the active virtual character due to the manner in which they interpreted the behavior of the virtual characters: "I think its probably the fact that there was no negotiation going on, I think friends tend to negotiate things even if they get angry, they tend to settle a negotiation. Whereas here, the role was one of the dominant and submission. (P4121r)". Some of these participants went further than simply attributing a higher status to the active virtual character. The participants reported deliberately choosing the active virtual character for interaction since the active virtual character looked commanding, more engaging and looked more approachable. Furthermore, three participants reported playing a passive role in their interactions with the active virtual character because they felt intimidated and negatively judged: "I think the guy on the right [active] looked at me but I think he remained quite distant and I had a sense he was kind of looking down to me (P4121r)." One participant felt that the active virtual character was "unimpressed" with their performance in the maze.

Participants attributed the passive character's lack of responsiveness to the social relationship between the two virtual characters rather than to technical constraints imposed by the VR system. This was especially the case when participants tried to interact with the passive character and were unsuccessful: "They were trying to get rid of me because they wanted to talk about what they were talking about. I think that is why I said they were trying to get rid of me. So one guy was instructing or telling the other guy off. And he was silent. It wasn't his place to talk to me. Because the other guy was taking control of the conversation and continued the conversation whenever I came (P2112)." Participants reported that the passive character was less friendly toward the participants due to the lack of interaction with the participants. Three participants felt that the passive virtual character was nonresponsive because of some unseen earlier problematic incidents it might have had with the active virtual character. Twenty-six participants reported not paying attention to the passive virtual character. This effect was observed throughout to such an extent that participants could not accurately describe the visual appearance of the passive virtual character. One participant did not even remember the gender of the passive virtual character: "The person on the right hand [active] tells me what I had to know and they go back to their little thing and the person on the left [passive] might have said something, you know as in "hi" or something like when I approached them but I don't think he or she gave me any directions at all. (P3121)". Six participants did not interact with the passive virtual character because the lack of responsiveness made the passive virtual character appear "indifferent" and "cold." However, 12 participants did not pay attention to the passive virtual character because the active virtual character took control of the interaction and appeared to be the virtual character in charge before the participant's interaction period with the virtual characters. Yet, the passive virtual character's behavior was not judged as being unrealistic. It was generally accepted that the behaviors portrayed by the passive virtual character for the role it played was natural and in keeping with the scenario: "It was always the one on the right [active] which was addressing me and the one on the left [passive] never said anything. So I guess, in that way I was responding as if they were real people because I expect if two people were having an argument and one was dominant, and then you came up to him and asked, you know for directions or something, it is going to be the one who is dominant in the argument that is going to say something (P7221)."

\section{Discussion}

The experiment was designed to ascertain the value of using meaningful postures in life-size virtual characters in addition to investigating the role of posture in the communication of affect. This was investigated through collecting responses of participants to sequences of behaviors exhibited by the virtual characters and examining the range and variation of these responses with the view to uncovering whether the responses were in keeping with those observed in the physical world. Analysis of the participant responses indicated that the Emotional postures designed to portray anger and sad in our experiment did not play an important role in the way participants respond to virtual characters. Our results indicate that it is better to utilize no postural cues as opposed to using incorrect postural cues.

Generally, a higher physiological arousal is expected to be associated with a higher reported presence. In the Angry conditions, neutral postures were positively associated with reported copresence and also positively associated with physiological arousal. Therefore, it was clear that the neutral postures outperformed the angry postures with respect to eliciting realistic participant responses. Similar to the Angry conditions, the neutral postures in the Sad conditions were also positively associated with reported copresence; however, they were negatively associated with physiological arousal. The normal association between physiological arousal and reported presence was not apparent in the Sad conditions. Therefore, analysis of the 
reported presence scores and physiological responses indicates that the Emotional postures adapted from Coulson [4] did not elicit responses similar to those observed in the physical world.

Yet, the analysis of the participant interviews and emotional state recognition questionnaire responses revealed that 11 out of the 14 participants, in the Angry conditions with postural cues, accurately recognized the emotional state of the active character as anger and reported that body language was a primary indicator of the active character's emotional state. Furthermore, in the conditions with congruent angry behavioral cues, all seven participants accurately recognized the underlying emotional state of the active character. However, the participant interviews and emotional state recognition questionnaire responses did not suggest that participants were able to accurately recognize the active character's emotional state in the Sad conditions.

This suggests that even though the parametric model for affective postures used in the Angry conditions portrayed postural cues associated with anger, however, the resulting behavior was incorrect. In the case of the Sad conditions, the results indicate that the parametric model of affective postures did not accurately portray cues associated with Sadness. One reason for the effects experienced may be in the underlying base postures used in the conditions. Eight out of the fourteen participants, in the Sad conditions with affective postures, attributed an emotional state of neutral to the active character, while a further four participants thought the active character was angry. Paterson et al. [53] suggest that body movement play a key role in the portrayal of emotional states. It is possible that the lack of cues in the quality of body movement, especially in the Sad conditions, prevented participants from accurately recognizing the postures.

In keeping with observations in the physical world, participants maintained social norms in relation to spatial behavior as measured through the tracking data and as reported in the participant interviews. Surprisingly, the different experiment conditions did not seem to have had an effect on the social distances maintained by the participants with the characters. For instance, it was reasonable to expect that a participant approaching an angry character might be fearful or apprehensive and, therefore, maintain larger interpersonal distances with the character, while a participant approaching a sad character might be sympathetic and, therefore, maintain smaller interpersonal distances with the character. However, no significant differences were detected in the analysis of the tracking data. It is possible that the participants' perception of the characters did not affect the interpersonal distances maintained by the participant because of the nature of the task. Another reason for the lack of factor-related differences could be due to the mechanism used to trigger the characters into turning to focus attention toward the participant. Independent of condition, the characters were triggered to stop their conversation and turn toward the participant at the same distance. This could in turn be the factor that controlled the distance at which participants interact with the character. Furthermore, a number of realistic responses were reported by the participants in the participant interviews including feelings of intrusion and the desire to maintain acceptable social norms with the characters.

Results uncovered from the participant interviews suggest that context plays an important role in the participant's judgement of the virtual characters. Although participants could not understand the contents of the conversation, the quality of the conversation was essentially neutral and emotionless. It is possible that participants found it easier to attribute angry connotations to a neutral conversation as opposed to sad ones. This is in keeping with the better accuracy in the participant's ability to judge the virtual character's emotional state. It is also possible that the qualities of the verbal conversation between the characters were not in keeping with a Sad scenario. This could account for the reduced success in accurately recognizing the emotional states of the virtual characters in the Sad conditions. In addition, the muffled conversation may have played a role in allowing participants to project their perceptions onto the characters when the characters displayed neutral postural cues. Participant interviews suggested that the Emotional postures played a crucial role in preventing participants from projecting their perceptions about the characters onto the scenario, while the neutral postures seem to allow the participants to interpret the scenario as they saw fit. In other words, it appears that neutral postures fitted in better within the context than the Emotional postures designed to portray anger.

In addition to context, results from the participant interviews suggest that it is important to design the social scene in virtual environments in a holistic manner. Many participants remarked on the appearance of the characters by referring to the active character as having authority over the passive character. In the Angry conditions, with Emotional postures, the participants continued to infer that the passive character was being chastised due to some blunder on his part. They refer to the body language of the active character as being aggressive and agitated toward the passive character. These participants also referred to the conversation between the virtual characters as an "argument." This suggests that participant's perception of the active character was influenced by their perception of the whole social relationship between the two characters. In other words, since participants perceived the situation as an argument, if one character was angry then the other, by implication, should have been afraid. In the Neutral and Sad conditions, references to the body language of the characters indicating an argument were rarely made; however, the active character was at times judged as being angry. In these instances, the passive character was judged as either afraid or neutral. This suggests that it is equally important to design appropriate behavioral cues for the passive character as it is for the active character since the behavior of the passive character influences the participant's interpretation of the active character's emotional state.

Possible explanations for the results from the selfreported copresence scores and physiological responses were obtained from the participant interviews and affect recognition questionnaires. Our analysis of the participant responses did not support our hypothesis; however, the usage of a variety of data-collection methods provided 
interesting insights and possible avenues for future research. These were gained purely through analyzing the participant interviews. These insights included the observation that participants attributed a wide variety of perceived emotional states or attitudes to the characters and were able to interpret the social context presented to them with many rich details. This validates the use of mixed methods to design, develop, and evaluate models of behavioral cues for use in expressive virtual characters. Generally, the method calls for equal priority to be given to all the collected data, but in this case, the qualitative results were used mainly to strengthen the quantitative results or provide explanations for discrepancies. This approach fitted neatly into both the operational definition of presence and the research aims of the experiment. The use of multiple participant responses to evaluate interactions with virtual characters has the potential to give a more concrete understanding of the interaction between participants and virtual characters. Furthermore, in the case of an exploratory investigation, the additional use of semistructured interviews can be a powerful way in which to investigate participant responses to virtual characters.

\section{ACKNOWLEDGMENTS}

The authors would like to thank Andrea Brogni, Marco Gillies, David Swapp, and Mette Thomsen for their help throughout the project. This work was supported through the EQUATOR Interdisciplinary Research Collaboration (EPSRC Grant GR/N15986/01).

\section{REFERENCES}

[1] E. Arts, The Sims Online, http://thesims.ea.com/, Jan. 2005.

[2] K. Johnsen, R. Dickerson, A. Raij, B. Lok, J. Jackson, M. Shin, J. Hernandez, A. Stevens, and D.S. Lind, "Experiences in Using Immersive Virtual Characters to Educate Medical Communication Skills," Proc. IEEE Virtual Reality Conf. (VR '05), vol. 324, pp. 179186, 2005.

[3] D.-P. Pertaub, M. Slater, and C. Barker, "An Experiment on Public Speaking Anxiety in Response to Three Different Types of Virtual Audience," Presence: Teleoperators and Virtual Environments, vol. 11, no. 1, pp. 68-78, Feb. 2002.

[4] M. Coulson, "Attributing Emotion to Static Body Postures: Recognition Accuracy, Confusions, and Viewpoint Dependence," J. Nonverbal Behavior, vol. 28, no. 2, pp. 117-139, 2004.

[5] B. de Gelder, "Towards the Neurobiology of Emotional Body Language," Nature Rev. Neuroscience, vol. 7, pp. 242-249, 2006.

[6] V. Vinayagamoorthy, M. Gillies, A. Steed, E. Tanguy, X. Pan, C. Loscos, and M. Slater, "Building Expression into Virtual Characters," Proc. Eurographics '06, State of the Art Report (STAR), pp. 21-61, Sept. 2006.

[7] V. Vinayagamoorthy, M. Garau, A. Steed, and M. Slater, "An Eye Gaze Model for Dyadic Interaction in an Immersive Virtual Environment: Practice and Experience," Computer Graphics Forum, vol. 23, no. 1, pp. 1-11, Mar. 2004.

[8] V. Vinayagamoorthy, A. Brogni, A. Steed, and M. Slater, "The Influence of a Virtual Character Posture Model on Reported Presence in an Immersive Virtual Environment," Proc. ACM SIGGRAPH Int'l Conf. Virtual Reality Continuum and Its Applications (VRCIA '06), pp. 229-236, June 2006.

[9] M.V. Sanchez-Vives and M. Slater, "From Presence to Consciousness through Virtual Reality," Nature Rev. Neuroscience, vol. 6, no. 4, pp. 332-339, 2005.

[10] J. Blascovich, J. Loomis, A.C. Beall, K. Swinth, C. Hoyt, and J.N. Bailenson, "Immersive Virtual Environment Technology as a Methodological Tool for Social Psychology," Psychology Inquiry, vol. 13, pp. 103-124, 2002.
[11] J. Gratch and S. Marsella, "A Domain Independent Framework for Modeling Emotion," J. Cognitive Systems Research, vol. 5, no. 4, pp. 269-306, 2004.

[12] E.A.R. Tanguy, "Emotions: The Art of Communication Applied to Virtual Actors," PhD dissertation, June 2006.

[13] S. Planalp, V.L. DeFrancisco, and D. Rutherford, "Varieties of Cues to Emotion in Naturally Occurring Situations," Cognition and Emotion, vol. 10, no. 2, pp. 137-153, 1996.

[14] R. Schroeder, The Social Life of Avatars; Presence and Interaction in Shared Virtual Environments. Springer-Verlag New York, Inc., 2002.

[15] M. Argyle, Social Interaction. Tavistock Publications, 1969.

[16] P. Ekman, "Facial Expressions of Emotion: New Findings, New Questions," Psychological Science, vol. 3, no. 1, pp. 34-38, 1992.

[17] S. Kshirsagar and N. Magnenat-Thalmann, "A Multilayer Personality Model," Proc. Second Int'l Symp. Smart Graphics, pp. 107-115, June 2002.

[18] I. Albrecht, M. Schröder, J. Haber, and H.-P. Seidel, "Mixed Feelings: Expression of Non-Basic Emotions in a Muscle-Based Talking Head," Special Issue of J. Virtual Reality on Language, Speech and Gesture, vol. 8, pp. 201-212, 2005.

[19] P. Ekman and V.W. Friesen, "Head and Body Cues in the Judgement of Emotion: A Reformulation," Perceptual and Motor Skills, vol. 24, pp. 711-724, 1967.

[20] M. Argyle, Bodily Communication, second ed. Methuen, 1998.

[21] P.E. Bull, Posture and Gesture, ser. Int'l Series in Experimental Social Psychology, vol. 16, Pergamon Press, 1987.

[22] K.L. Walters and R.D. Walk, "Perception of Emotion from Body Posture," Bull. Psychonomic Soc., vol. 24, no. 5, p. 329, 1986.

[23] A.T. Dittmann, M.B. Parloff, and D.S. Boomer, "Facial and Bodily Expression: A Study of Receptivity of Emotional Cues," Psychiatry, vol. 28, pp. 239-244, 1965.

[24] A.E. Scheflen, "The Significance of Posture in Communication Systems," Psychiatry, vol. 27, pp. 316-331, 1964.

[25] K.L. Walters and R.D. Walk, "Perception of Emotion from Moving Body Cues in Photographs," Bull. Psychonomic Soc., vol. 26, no. 2, pp. 112-114, 1988.

[26] W.T. James, "A Study of the Expression of Bodily Posture," J. General Psychology, vol. 42, pp. 90-94, 1932.

[27] H.G. Wallbott, "Bodily Expression of Emotion," European J. Social Psychology, vol. 28, no. 6, pp. 879-896, 1998.

[28] P.R. De Silva and N. Bianchi-Berthouze, "Modeling Human Affective Postures: An Information Theoretic Characterization of Posture Features: Research Articles," Computer Animation and Virtual Worlds, vol. 15, no. 3-4, pp. 269-276, 2004.

[29] A. Kelsmith, R.P. De Silva, and N. Bianchi-Berthouze, "Grounding Affective Dimensions into Posture Features," Proc. First Int'l Conf. Affective Computing and Intelligent Interaction (ACII '05), ser. Lecture Notes in Computer Science, J. Tao, T. Tan, and R.W. Picard, eds., vol. 3784, pp. 263-270, Oct. 2005.

[30] M. Slater, "How Colourful Was Your Day?: Why Questionnaires Cannot Assess Presence in Virtual Environments," Presence: Teleoperators and Virtual Environments, vol. 13, no. 4, pp. 484-493, 2004.

[31] J.N. Bailenson, J. Blascovich, and A.C. Beall, “Equilibrium Theory Revisited: Mutual Gaze and Personal Space in Virtual Environments," Presence: Teleoperators and Virtual Environments, vol. 10, no. 6, pp. 583-598, 2001.

[32] M. Meehan, B. Insko, M.C. Whitton, and F.P. Brooks, "Physiological Measures of Presence in Stressful Virtual Environments," Proc. ACM SIGGRAPH '02, vol. 21, pp. 645-653, 2002.

[33] M. Slater, C. Guger, G. Edlinger, R. Leeb, G. Pfurtscheller, A. Antley, M. Garau, A. Brogni, A. Steed, and D. Friedman, "Analysis of Physiological Responses to a Social Situation in an Immersive Virtual Environment," Presence: Teleoperators and Virtual Environments, vol. 15, no. 5, Oct. 2006

[34] E.T. Hall, The Hidden Dimension: Man's Use of Space in Public and Private. Bodley Head, 1969.

[35] M.J. Green, L.M. Williams, and D. Davidson, "In the Face of Danger: Specific Viewing Strategies for Facial Expressions of Threat," Cognition and Emotion, vol. 17, no. 5, pp. 779-786, 2003.

[36] C. Cruz-Neira, D.J. Sandin, and T.A. DeFanti, "Surround-Screen Projection-Based Virtual Reality: The Design and Implementation of the Cave," Proc. ACM SIGGRAPH '93, pp. 135-142, 1993.

[37] A. Steed, J. Mortensen, and E. Frécon, "Spelunking: Experiences Using the Dive System on Cave-Like Platform," Immersive Projection Technologies and Virtual Environments, pp. 153-164, 2001. 
[38] R.M. Taylor II, T.C. Hudson, A. Seeger, H. Weber, J. Juliano, and A.T. Helser, "VRPN: A Device-Independent, Network-Transparent VR Peripheral System," Proc. ACM Symp. Virtual Reality Software and Technology (VRST '01), pp. 55-61, 2001.

[39] M. Gillies, V. Vinayagamoorthy, D. Robertson, and A. Steed, "A Platform Independent Architecture for Virtual Characters and Avatars," Proc. Third Int'l Workshop Intelligent Virtual Agents (IVA '05), T. Panayiotopoulos, J. Gratch, R. Aylett, D. Ballin, P. Olivier, and T. Rist, eds., vol. 3661, Sept. 2005.

[40] M.P. Johnson, "Exploiting Quaternions to Support Expressive Interactive Character Motion," PhD dissertation, Feb. 2003.

[41] P. Ekman, Emotion in the Human Face, vol. 2, Cambridge Univ. Press, 1982.

[42] M. Garau, M. Slater, V. Vinayagamoorthy, A. Brogni, A. Steed, and A.M. Sasse, "The Impact of Avatar Realism and Eye Gaze Control on the Perceived Quality of Communication in a Shared Immersive Virtual Environment," Proc. SIGCHI Conf. Human Factors in Computing Systems (CHI '03), pp. 529-536, Apr. 2003.

[43] M. Slater and A. Steed, "A Virtual Presence Counter," Presence: Teleoperators and Virtual Environments, vol. 9, no. 2, pp. 214-217, 2000.

[44] D. Watson and R. Friend, "Social Avoidance and Distress Scale [SAD] (1969)," Measures for Clinical Practice: A Sourcebook, K. Corcoran and J. Fischer, eds., Free Press, 1987.

[45] C.D. Spielberger, R.L. Gorsuch, R. Lushene, P.R. Vagg, and G.A Jacobs, Manual for the State-Trait Anxiety Inventory. Consulting Psychologists Press, 1983.

[46] T. Gilbert and M. Coulson, "The Role of Emotional Perception in Attentional Bias for Threat," Proc. British Psychological Soc., vol. 10, no. 2, p. 68, 2002.

[47] W. Doherty, "The Emotional Contagion Scale: A Measure of Individual Differences," J. Nonverbal Behaviour, vol. 21, no. 2, pp. 131-154, 1997.

[48] P. McCullagh and J.A. Nelder, Generalized Linear Models, second ed. Chapman and Hall, 1989.

[49] Generalised Linear Interactive Model (GLIM), T.N.A.G. NAG, http:// www.nag.co.uk/stats/gdge_soft.asp/, July 2005.

[50] A. Tashakkori and C. Teddlie, Mixed Methodology: Combining Qualitative and Quantitative Approaches, ser. Applied Social Research Methods Series, vol. 46, Sage Publications, 1998

[51] Atlas.ti-The Knowledge Workbench, ATLAS.ti, http://www.atlasti. com/index.php, Mar. 2006.

[52] J.L. Andreassi, Psychophysiology: Human Behavior and Physiological Response. Lawrence Erlbaum Assoc., 1995.

[53] H.M. Paterson, F.E. Pollick, and A.J. Sanford, "The Role of Velocity in Affect Discrimination," Proc. 23rd Ann. Conf. Cognitive Science Soc. (CSS '01), J. D. Moore and K. Stenning, eds., pp. 756761, Aug. 2001.

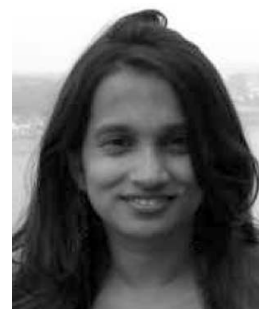

Vinoba Vinayagamoorthy received the degree in information systems engineering from the University of Surrey, Guildford, UK, in 2001 and the doctoral degree from the University College London (UCL), in 2006. Her thesis is on "Participant Responses to Virtual Agents in Immersive Virtual Environments." She works in Research and Innovation at the British Broadcasting Corporation (BBC). Her interests include computer graphics, virtual reality, mixed reality, 3DTV, virtual characters, and human-computer interaction. Prior to joining BBC, she worked as a research fellow at UCL between 2001 and 2007. Her work has appeared in a range of publications including ACM SIGCHI, ACM VRST, and Eurographics.

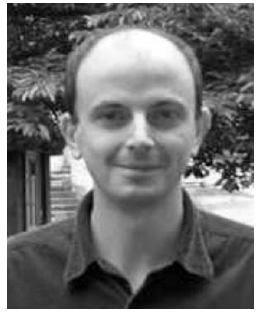

Anthony Steed is a reader in Virtual Environments at the University College London. He is the current head of the Virtual Environments and Computer Graphics group at UCL. His research interests include large-scale model rendering, immersion and presence in virtual environment displays, and interaction and collaboration between users of virtual environments systems. His recent work is published in several international journals and conferences including ACM VRST, IEEE VR, ACM SIGGRAPH, Human Factors, Presence: Teleoperators and Virtual Environments, IEEE Computer Graphics and Applications, and Computers and Graphics. He is coauthor with Slater of the textbook Computer Graphics and Virtual Environments: From Realism to Real-Time. He is a member of the IEEE.

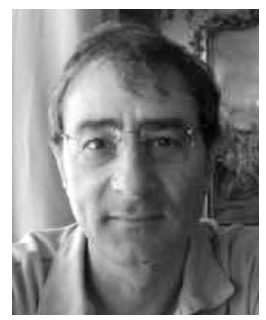

Mel Slater founded the Virtual Environments and Computer Graphics group at University College London. He has carried out research both on graphics rendering algorithms, a virtual light field approach to real-time global illumination, and also on understanding people's responses to their experiences in immersive virtual environments. In particular, he has pioneered research on presence in virtual environments, including its application to psychotherapy, and recently coauthored a paper in Nature Reviews Neuroscience that connects this topic with research on perception and consciousness. Since January 2006, he has been leading a new group on presence research and interaction as an ICREA research professor at the Virtual Reality Centre of Barcelona, Universitat Politècnica de Catalunya, Spain.

$\triangleright$ For more information on this or any other computing topic, please visit our Digital Library at www.computer.org/publications/dlib. 